\title{
DE-Sinc Method for Second Order Singularly Perturbed Boundary Value Problems*
}

\author{
Masatake Mori, Ahniyaz Nurmuhammad ${ }^{\dagger, \ddagger}$ and Mayinur MuhammaD ${ }^{\S}$ \\ Department of Mathematical Sciences \\ Tokyo Denki University \\ Hatoyama, Saitama 350-0394, Japan \\ †E-mail: nur@r.dendai.ac.jp
}

Received December 3, 2007

Revised June 5, 2008

\begin{abstract}
In this paper the sinc-Galerkin method, as well as the sinc-collocation method, based on the double exponential transformation (DE transformation) for singularly perturbed boundary value problems of second order ordinary differential equation is considered. A large merit of the present method exists in that we can apply the standard sinc method with only a small care for perturbation parameter. Through several numerical experiments we confirmed higher efficiency of the present method than that of other methods, e.g., sinc method based on the single exponential (SE) transformation, as the number of sampling points increases.
\end{abstract}

Key words: double exponential transformation, DE formula, DE-sinc method, singularly perturbed problem

\section{Introduction}

Singular perturbations appear in various fields of science and engineering. It is known that these problems depend on a small positive parameter $\varepsilon$ in such a way that the solution exhibits a multiscale character, i.e., there are thin layers where the solution changes rapidly, while away from the layers it behaves regularly. And hence numerical solution of singularly perturbed problems usually presents difficulties that we have to be careful when choosing numerical method. There exist a variety of methods for solving singularly perturbed boundary value problems, such as methods based on the asymptotic analysis, methods based on the non-asymptotic analysis called boundary value technique [13] and methods based on the initial value technique [4]. As to research works on numerical solution of singularly perturbed boundary value problems see $[5,11,14]$ and references therein.

The idea of application of the sinc method to singularly perturbed boundary value problem is discussed by F. Stenger [15], and M. El-Gamel and J.R. Cannon [2] actually tried to solve numerically a second order singularly perturbed boundary value problem of ordinary differential equation using the sinc-Galerkin method. It is known that the sinc method usually gives a result with high accuracy even

\footnotetext{
* Partially supported by the Grant-in-Aid for the 21st Century COE (Center of Excellence) Research by the Ministry of Education, Culture, Sports, Science and Technology, and also by the Grant-in-Aid for Scientific Research (C) by Japan Society for the Promotion of Science.

¥In Pinyin notation: Aheniyazi Nuermaimaiti.

$\S$ In Pinyin notation: Maimaiti Mayinuer.
} 
for problems with an algebraic singularity at the end point. They used the single exponential transformation (SE transformation, see Section 2) [7, 15] in order to transform the interval $(a, b)$ of the original problem onto the interval $(-\infty, \infty)$ on which sinc expansion is defined. It is known from the error analysis of the sinc method based on the SE transformation that error bound of the approximate solution is $O\left(\exp \left(-c^{\prime} \sqrt{N}\right)\right), c^{\prime}>0$, where $N$ is a parameter representing the number of terms in the sinc approximation. For more details about the sinc method see $[6,15]$.

On the other hand, high efficiency of the double exponential transformation (DE transformation) in numerical integration [16, 19] is well-known and it naturally suggests that DE transformation technique may be useful in other numerical methods. Indeed, it has been shown in $[8,18]$ that use of DE transformation technique in sinc methods results in highly efficient methods for numerical computation. For example, Sugihara applied sinc-collocation method based on the DE transformation to numerical solution of boundary value problems of second order ordinary differential equation and found that error bound of the method is $O(\exp (-c N / \log N)), c>0[17]$ which converges to zero much faster than that of the method based on the SE transformation as $N$ becomes large. Also, Nurmuhammad et al. employed the sinc-collocation [9] and the sinc-Galerkin [10] methods based on the DE transformation for solving boundary value problems of fourth-order ordinary differential equation and found that the results always exhibit error behavior $O(\exp (-c N / \log N))$ as long as the DE transformation is used in the sinc method $[9,10]$.

The aim of the present paper is to propose a sinc-Galerkin method based on the DE transformation which we call the DE sinc-Galerkin method, as well as a sinc-collocation method which we call the DE sinc-collocation method, to solve the second order singularly perturbed boundary value problem of ordinary differential equation with homogeneous boundary condition

$$
\begin{aligned}
& \varepsilon y^{\prime \prime}(x)+\mu_{1}(x) y^{\prime}(x)+\mu_{0}(x) y(x)=\sigma(x), \quad a<x<b, \\
& y(a)=y(b)=0,
\end{aligned}
$$

where $\varepsilon$ is a small positive constant called perturbation parameter. Throughout the present paper we assume that $\mu_{1}(x), \mu_{0}(x)$ and $\sigma(x)$ are analytic on $a<x<b$, and also that the solution $y(x)$ exists and is analytic on $a<x<b$.

We apply the DE sinc-Galerkin method also to the semi-linear problem, and we also propose a sinc-collocation method based on the DE transformation to the present singularly perturbed problem for comparison with the sinc-Galerkin method.

The main point of our method exists in the sinc expansion based on the DE transformation and in the DE quadrature formula [19] for approximation of the inner product appearing in the sinc-Galerkin method. The reason why we employ the DE transformation for numerical solution of singularly perturbed problems is as follows. One of the remarkable characteristics of the DE transformation, as well as that of the SE transformation, is that sampling points of the method 
based on the DE transformation accumulate to the boundary edge, and hence the method in itself can get sufficient information in the boundary layer. Indeed, in the sinc method there are infinite number of sampling points in the boundary layer from its nature and we can choose as many sampling points as we require in the neighborhood of the boundary edge when we actually carry out numerical computation. Also, because of such a distribution of sampling points, the present DE-sinc method usually gives a result with high accuracy even for a problem which has both a boundary layer as well as an algebraic singularity at the boundary edge. See Example 4 in Section 7.

\section{Sinc expansion and the DE transformation}

As is well known [15] sinc function is defined on $-\infty<t<\infty$

$$
\operatorname{sinc}(t)= \begin{cases}\frac{\sin \pi t}{\pi t} ; & t \neq 0 \\ 1 ; & t=0\end{cases}
$$

We fix mesh size $h$ and employ a set which consists of sinc functions

$$
S(j, h)(t)=\frac{\sin \frac{\pi}{h}(t-j h)}{\frac{\pi}{h}(t-j h)}, \quad j=0, \pm 1, \pm 2, \ldots
$$

as the basis for the numerical solution of the present problem. It is easy to see that it satisfies an orthogonal relation

$$
S(j, h)(k h)=\operatorname{sinc}(k-j)= \begin{cases}1 ; & k=j, \\ 0 ; & k \neq j .\end{cases}
$$

Now we consider a function $u(t)$ defined on $-\infty<t<\infty$. We assume here that i) $\quad u(t)$ is analytic on a strip domain

$$
|\operatorname{Im} t|<d
$$

in the $t$-plane,

ii) $|u(t)| \rightarrow 0$ as $t \rightarrow \pm \infty$.

Then, if the series

$$
u_{h}(t)=\sum_{j=-\infty}^{\infty} u(j h) S(j, h)(t)
$$

converges we call it Whittaker cardinal expansion. Note that $u_{h}(t)$ is an interpolation to $u(t)$ because

$$
u_{h}(k h)=u(k h)
$$


holds from (2.3). Under assumptions (i) and (ii) given above with some additional mild conditions we can write [15]

$$
u(t)=u_{h}(t)+E_{\text {sinc }}(h), \quad E_{\text {sinc }}(h)=O\left(\exp \left(-\frac{\pi d}{h}\right)\right),
$$

where $d$ is the half width of the strip domain defined in (2.4). Since the error $E_{\text {sinc }}(h)$ is due to discretization of $u(t)$ we call it discretization error. We see that as $h$ becomes small $E_{\text {sinc }}(h)$ decreases very quickly.

Sinc expansion (2.7) is defined on $-\infty<t<\infty$, while the equation we are going to deal with is defined on the finite interval $a<x<b$, and hence we need some transformation which maps $a<x<b$ onto $-\infty<t<\infty$. In many of applications of the sinc method transformation

$$
x=\psi(t)=\frac{b-a}{2} \tanh \frac{t}{2}+\frac{b+a}{2}
$$

has been used $[2,15]$. On the other hand, we employ here transformation

$$
x=\psi(t)=\frac{b-a}{2} \tanh \left(\frac{\pi}{2} \sinh t\right)+\frac{b+a}{2}
$$

which Takahasi and Mori proposed for numerical integration in 1974 [19]. Note that inverse transformation to (2.9) can be written explicitly

$$
t=\psi^{-1}(x)=\phi(x)=\log \left(\frac{1}{\pi} \log \frac{x-a}{b-x}+\sqrt{\left(\frac{1}{\pi} \log \frac{x-a}{b-x}\right)^{2}+1}\right) .
$$

One of the main reasons why we use it is that (2.9) is an optimal transformation in some sense and hence in the sinc method it usually gives significantly faster convergence than $(2.8)[16,18,19]$.

We substitute $t$ in $(2.7)$ with $\psi^{-1}(x)$ from $(2.10)$ and write

$$
v(x)=u\left(\psi^{-1}(x)\right) .
$$

Then from (2.5) we obtain an approximation to the function $v(x)$ defined on $a<x<b$

$$
\begin{aligned}
& v(x)=u\left(\psi^{-1}(x)\right)=v_{h}(x)+E_{\text {sinc }}(h), \\
& v_{h}(x)=\sum_{j=-\infty}^{\infty} v(\psi(j h)) S(j, h)\left(\psi^{-1}(x)\right), \\
& E_{\text {sinc }}(h)=O\left(\exp \left(-\frac{\pi d}{h}\right)\right) .
\end{aligned}
$$

It is known that for transformation (2.9) $d$ is a positive number satisfying $d \leq \frac{\pi}{2}$, while for transformation $(2.8) d$ is a positive number satisfying $d \leq \pi$. 
Corresponding to homogeneous boundary condition $y(a)=y(b)=0$ in $(1.1)$ we generalize the order of zeros and assume that $y(x)$ satisfies

$$
y(x)= \begin{cases}O\left((x-a)^{\beta_{-}}\right) ; & x \rightarrow a\left(0<\beta_{-}\right), \\ O\left((b-x)^{\beta_{+}}\right) ; & x \rightarrow b\left(0<\beta_{+}\right)\end{cases}
$$

in the neighborhood of the boundary points.

Then, from (2.9) we have

$$
\left\{\begin{array}{l}
x-a=\frac{b-a}{1+\exp (-\pi \sinh t)} \approx(b-a) \exp \left(-\frac{\pi}{2} e^{|t|}\right) ; \quad t \rightarrow-\infty, \\
b-x=\frac{b-a}{1+\exp (+\pi \sinh t)} \approx(b-a) \exp \left(-\frac{\pi}{2} e^{t}\right) ; \quad t \rightarrow+\infty .
\end{array}\right.
$$

Therefore from (2.13) we see that $y(x)=y(\psi(t))$ decays in a double exponential way

$$
y(\psi(t))= \begin{cases}O\left(\exp \left(-\frac{\pi}{2} \beta_{-} \exp |t|\right)\right) ; & t \rightarrow-\infty, \\ O\left(\exp \left(-\frac{\pi}{2} \beta_{+} \exp t\right)\right) ; & t \rightarrow+\infty .\end{cases}
$$

In this sense we call (2.9) the double exponential transformation (DE transformation). On the other hand, in case of transformation $(2.8) y(x)=y(\psi(t))$ decays

$$
y(\psi(t))= \begin{cases}O\left(\exp \left(-\beta_{-}|t|\right)\right) ; & t \rightarrow-\infty, \\ O\left(\exp \left(-\beta_{+} t\right)\right) ; & t \rightarrow+\infty,\end{cases}
$$

so that we call it the single exponential transformation (SE transformation).

In the singularly perturbed problem we need to investigate behavior of $y(x)$ carefully in a very thin region at the boundary edge. For that purpose transformation (2.10) meets the requirement because, as is pointed out in Section 1, it enables us to use as many sampling points as we require in the close neighborhood of the boundary edge.

\section{Truncation of infinite summation}

In (2.12) we think of the solution $y(x)$ for $v(x)$ because we use sinc expansion for numerical solution over $(a, b)$. And hence we assume hereafter that $y(\psi(t))$ is analytic in a strip domain $|\operatorname{Im} t|<d$ for some $d$ corresponding to (2.4). From (2.12) we have

$$
\begin{aligned}
& y(x)=\sum_{j=-\infty}^{\infty} y\left(x_{j}\right) S(j, h)\left(\psi^{-1}(x)\right)+E_{\text {sinc }}(h), \\
& x_{j}=\psi(j h), \quad E_{\text {sinc }}(h)=O\left(\exp \left(-\frac{\pi d}{h}\right)\right),
\end{aligned}
$$


where each of $x_{j}=\psi(j h), j=0, \pm 1, \pm 2, \ldots$ is called sinc point. Then we replace $y\left(x_{j}\right)$ with its approximation $y_{j}$ and write

$$
\tilde{y}_{h}(x)=\sum_{j=-\infty}^{\infty} y_{j} S(j, h)\left(\psi^{-1}(x)\right) .
$$

In actual computation, however, we must truncate infinite sum (3.2) into a finite one. Suppose that we truncate sum (3.2) at $j=-n_{-}$on the negative side of $j$. Then, since we assume that $y(x)$ decays as $(2.15)$ and $|S(j, h)(t)| \leq 1$ from (2.1), the error due to truncation will be approximately bounded from above as

$$
\begin{aligned}
& \exp \left(-\frac{\pi}{2} \beta_{-} e^{\left(n_{-}+1\right) h}\right)+\exp \left(-\frac{\pi}{2} \beta_{-} e^{\left(n_{-}+2\right) h}\right)+\cdots \\
& =\delta^{e^{h}}+\delta^{e^{2 h}}+\cdots, \quad \delta=\exp \left(-\frac{\pi}{2} \beta_{-} e^{n_{-} h}\right) \\
& <\delta^{1+h}+\delta^{1+2 h}+\cdots=\delta \frac{\delta^{h}}{1-\delta^{h}}<\delta=\exp \left(-\frac{\pi}{2} \beta_{-} e^{n_{-} h}\right),
\end{aligned}
$$

where we assume that $\delta^{h}<1 / 2$ because $\delta$ is small [7]. Situation is the same when we truncate at $j=n_{+}$on the positive side of $j$.

We first review the standard strategy to truncate sinc expansion. For simplicity we assume here that $\beta_{-}=\beta_{+}=\beta$ and that $n_{-}=n_{+}=n$. Truncation of the sum should be carried out in such a way that error (3.3) due to truncation is equal to discretization error $\exp (-\pi d / h)$ due to sinc expansion (3.1), i.e.,

$$
\exp \left(-\frac{\pi}{2} \beta e^{n h}\right)=\exp \left(-\frac{\pi d}{h}\right)
$$

From this equation we obtain a relation between $h$ and $n$

$$
h=\frac{1}{n} \log \left(\frac{2 d n}{\beta}\right) .
$$

If we replace $h$ in $\exp (-\pi d / h)$ with $(3.5)$ we have the well-known form of error expressed in terms of $n$ [18]:

$$
E_{\mathrm{DE}}\left(=E_{\mathrm{sinc}}(h)\right)=O\left(\exp \left(-\frac{n}{\log (2 d n / \beta)}\right)\right) .
$$

Similarly, in case of the SE transformation (2.8) we have

$$
E_{\mathrm{SE}}=O\left(\exp \left(-\sqrt{\pi^{2} d \beta n / 2}\right)\right)
$$

if we choose $h=\sqrt{2 d / n}$. If we compare (3.6) with (3.7) we see that convergence by the DE transformation as $n$ becomes large is much faster than that by the $\mathrm{SE}$ transformation. In fact it is proved that DE-sinc approximation is optimal in some 
sense in the sinc approximation $[16,18]$. This is one of the significant reasons why we employed the DE transformation in the present method.

However, from the standpoint of applications, it is usually difficult to know in advance the value of $d$, half width of the domain defined in $(2.4)$ in which $y(\psi(t))$ is analytic. Therefore, we propose here another strategy which does not require the value of $d$ in advance instead of the standard strategy stated above.

Usually it is reasonable to assume that the maximum value of $|y(x)|$ in applications is of order 1 and hence we assume here that $\max _{0 \leq x \leq 1}|y(x)|$ is of order 1 . Note that $y(x)=y(\psi(t))$ decays as (2.15). Then, the strategy we propose here is as follows. First we select a small positive number $\varepsilon_{\text {tr }}$ for truncation parameter. Next we select mesh size $h$. Then, if $\left|y\left(x_{j}\right)\right|$ satisfies

$$
\left|y\left(x_{j}\right)\right| \leq \varepsilon_{\text {tr }} \quad \text { at } j=-n_{-}
$$

we regard that $y\left(x_{j}\right)$ has decayed sufficiently small at $j=-n_{-}$on the negative side of $j$ and stop summation (3.1) at the lower limit $j=-n_{-}$. That is, we set

$$
\exp \left(-\frac{\pi}{2} \beta_{-} e^{t_{-}}\right)=\varepsilon_{\mathrm{tr}}, \quad t_{-}=n_{-} h
$$

because $|y(x)|=|y(\psi(t))|$ is assumed to decay as (2.15). $-t_{-}$is the lower limit of truncation in variable $t$. This gives a relation between mesh size $h$ and number $n_{-}$ of terms in the sinc expansion of the solution

$$
n_{-}=\frac{1}{h} t_{-}, \quad t_{-}=\log \left(\frac{2}{\pi \beta_{-}} \log \frac{1}{\varepsilon_{\mathrm{tr}}}\right) .
$$

Similarly, if $\left|y\left(x_{j}\right)\right|$ satisfies $\left|y\left(x_{j}\right)\right| \leq \varepsilon_{\text {tr }}$ at $j=n_{+}$on the positive side of $j$, we stop summation at the upper limit $j=n_{+}$where

$$
n_{+}=\frac{1}{h} t_{+}, \quad t_{+}=\log \left(\frac{2}{\pi \beta_{+}} \log \frac{1}{\varepsilon_{\mathrm{tr}}}\right),
$$

and $t_{+}$is the upper limit of truncation in variable $t$.

Choice of $\varepsilon_{\text {tr }}$ depends on user's requirement for error tolerance and there are various ways for its choice. A very easy and general way is to select machine epsilon of the system on which numerical solution is computed for $\varepsilon_{\text {tr }}$ because a very simple program for computation of machine epsilon or its good approximation is known [3]. Actually, in the numerical examples given in Section 7 we select for $\varepsilon_{\text {tr }}$ machine epsilon of the system. In any case we should select $\varepsilon_{\text {tr }}$ equal to or larger than machine epsilon of the system.

So far we have not taken into consideration that $\varepsilon$, perturbation parameter, is very small. If we should be careful for smallness of $\varepsilon$ we can alternatively employ the following strategy. 
In singularly perturbed problem (1.1) we assume that $a=0$ and $b=1$ for simplicity. In the following we assume that $\mu_{1}(x)$ and $\mu_{0}(x)$ satisfy

$$
\mu_{1}(x)=0, \quad \mu_{0}(x) \leq-\alpha<0(\alpha>0) .
$$

Then from $[5,11]$ we see that boundary layers appear at both edge points $x=0$ and $x=1$, and that solution $y(x)$ satisfying homogeneous boundary condition $y(0)=0$ includes a component $y_{-}(x)$ which dominates in the left boundary layer and has a bound $\hat{y}_{-}(x)$ such that

$$
|y(x)| \approx\left|y_{-}(x)\right| \leq \hat{y}_{-}(x), \quad \hat{y}_{-}(x)=C_{-}\left|\exp \left(-\alpha \frac{x}{\sqrt{\varepsilon}}\right)-1\right| .
$$

In the singularly perturbed problem we assume that $\sqrt{\varepsilon}$ is itself a very small constant. However, at the left edge point $x=0$ we need to investigate behavior of $y(x)$ in a further thinner region $x \ll \sqrt{\varepsilon}$. In such a thin region $\hat{y}_{-}(x)=$ $C_{-}|\exp (-\alpha x / \sqrt{\varepsilon})-1| \approx C_{-} \alpha x / \sqrt{\varepsilon}$ holds. Therefore, in the close neighborhood of the left edge point $x=0$ we can write $\hat{y}_{-}(x) \approx C_{-} L_{-} x$ where $L_{-}(=\alpha / \sqrt{\varepsilon})$ is a large positive constant. Situation is the same in the close neighborhood of the right edge point $x=1$ and we can write $\hat{y}_{+}(x) \approx C_{+} L_{+}(1-x)$ where $L_{+}$is a large positive constant.

Now we assume that in such an extremely close neighborhood of the left edge point bound $\hat{y}_{-}(x)$ well approximates absolute value of the solution $|y(x)|$, so that we can carry out truncation of summation (3.2) based on the bound $\hat{y}_{-}(x) \approx L_{-} x$ (we neglected $C_{-}$) instead of the left side of (3.9), i.e., we set

$$
L_{-} \exp \left(-\frac{\pi}{2} \beta_{-} e^{t_{-}}\right)=\varepsilon_{\mathrm{tr}}, \quad t_{-}=n_{-} h
$$

because $x$ decays as (2.14). Then we have

$$
n_{-}=\frac{1}{h} t_{-}, \quad t_{-}=\log \left(\frac{2}{\pi \beta_{-}} \log \frac{L_{-}}{\varepsilon_{\mathrm{tr}}}\right)
$$

and, similarly,

$$
n_{+}=\frac{1}{h} t_{+}, \quad t_{+}=\log \left(\frac{2}{\pi \beta_{+}} \log \frac{L_{+}}{\varepsilon_{\mathrm{tr}}}\right) .
$$

In Examples 1, 2 and 3 in Section 7 we use (3.15) and (3.16).

We should note that $L_{-}$and $L_{+}$are used only to determine truncation point of the sinc expansion and do not appear in the algorithm of numerical solution. In addition $L_{-}$appears inside double logarithm in $n_{-}$in the right side of (3.15), so that effect of $L_{-}$on $n_{-}$or on $t_{-}$is very small. Situation is the same for $L_{+}$. See change of $t_{-}$and $t_{+}$in Table 1 in Example 1, for example. And hence, their influence to accuracy of numerical approximation is small. 
What we should emphasize is that the present sinc method based on the DE transformation is by itself capable of solving singularly perturbed problems even without paying particular care for the abnormal situation that $\varepsilon$ is very small. And hence, if it is difficult to select a suitable value of $L_{-}$we recommend to use $L_{-}=1$ as an alternative value which will give a useful result in many cases. Situation is the same for $L_{+}$. See Example 3 and 4 in Section 7 .

\section{DE sinc-Galerkin method}

Now we are ready to apply the Galerkin method for numerical solution. For the moment we generalize coefficient of the second derivative in the form of a function $\mu_{2}(x)$ instead of the constant $\varepsilon$ and consider the equation with homogeneous boundary condition

$$
\begin{aligned}
& \mu_{2}(x) y^{\prime \prime}(x)+\mu_{1}(x) y^{\prime}(x)+\mu_{0}(x) y(x)=\sigma(x), \quad a<x<b, \\
& y(a)=y(b)=0 .
\end{aligned}
$$

We assume here that $\mu_{2}(x)$ is analytic on $a<x<b$ as well as $\mu_{1}(x), \mu_{0}(x)$ and $\sigma(x)$ in (1.1).

We first introduce an inner product with a weight function $1 / \phi^{\prime}(x)=$ $1 /\left\{\psi^{-1}(x)\right\}^{\prime}$

$$
\langle f, g\rangle=\int_{a}^{b} f(x) g(x) \rho(x) d x, \quad \rho(x)=\frac{1}{\phi^{\prime}(x)} .
$$

Note that

$$
\begin{aligned}
\frac{1}{\phi^{\prime}(x)} & =\frac{1}{\frac{d \psi^{-1}(x)}{d x}}=\frac{d \psi(t)}{d t}=\frac{b-a}{2} \frac{\frac{\pi}{2} \cosh t}{\cosh ^{2}\left(\frac{\pi}{2} \sinh t\right)} \\
& \approx \pi \frac{b-a}{2} e^{|t|} \exp \left(-\frac{\pi}{2} e^{|t|}\right), \quad t \rightarrow \pm \infty
\end{aligned}
$$

holds. Then, the inner product of equation (4.1) and the sinc function

$$
S_{k} \equiv S(k, h)\left(\psi^{-1}(x)\right)
$$

gives a weak equation

$$
\left\langle\mu_{2} y^{\prime \prime}, S_{k}\right\rangle+\left\langle\mu_{1} y^{\prime}, S_{k}\right\rangle+\left\langle\mu_{0} y, S_{k}\right\rangle=\left\langle\sigma, S_{k}\right\rangle .
$$

Integration by parts on each term on the left hand side gives

$$
\left\langle\mu_{2} y^{\prime \prime}, S_{k}\right\rangle=\int_{a}^{b} y\left(\mu_{2} S_{k}\right)^{\prime \prime} d x+F_{2}, \quad F_{2}=\left.\left\{y^{\prime}\left(\mu_{2} S_{k} \rho\right)-y\left(\mu_{2} S_{k} \rho\right)^{\prime}\right\}\right|_{a} ^{b}
$$

and

$$
\left\langle\mu_{1} y^{\prime}, S_{k}\right\rangle=-\int_{a}^{b} y\left(\mu_{1} S_{k}\right)^{\prime} d x+F_{1}, \quad F_{1}=\left.\left\{y\left(\mu_{1} S_{k} \rho\right)\right\}\right|_{a} ^{b} .
$$


We assumed that $y(x)$ vanishes at the boundary as (2.13) and hence $F_{1}$ and the second term of $F_{2}$ vanish there. On the other hand, $y^{\prime}(x)$ may not vanish at the boundary as seen from (2.13). However, we defined the weight function $\rho(x)$ as (4.2) and hence from (4.3) we see that the first term of $F_{2}$ also vanishes at the boundary. And we finally have a weak form corresponding to the equation (4.1) without derivatives of $y(x)$ as follows:

$$
\int_{a}^{b} y\left(\mu_{2} S_{k} \rho\right)^{\prime \prime} d x-\int_{a}^{b} y\left(\mu_{1} S_{k} \rho\right)^{\prime} d x+\int_{a}^{b} y\left(\mu_{0} S_{k} \rho\right) d x=\int_{a}^{b} \sigma S_{k} \rho d x .
$$

Here we carry out numerical integration at each term of (4.8). For that purpose we employ the double exponential formula (DE formula, or DE quadrature formula) [19]

$$
\begin{aligned}
& \int_{a}^{b} f(x) d x=\int_{-\infty}^{\infty} f(\psi(t)) \psi^{\prime}(t) d t=h \sum_{j=-n_{-}}^{n_{+}} f\left(x_{j}\right) \frac{1}{\phi^{\prime}\left(x_{j}\right)}+E_{\mathrm{int}}+E_{\mathrm{trunc}} \\
& x_{j}=\psi(j h), \quad E_{\mathrm{int}}=O\left(\exp \left(-\frac{2 \pi d^{\prime}}{h}\right)\right)
\end{aligned}
$$

based on the DE transformation (2.9) with the same mesh size $h$ as the one we employed for sinc expansion (3.1), i.e., the set of the sinc points $x_{j}=\psi(j h)$, $j=0, \pm 1, \pm 2, \ldots$ is common to the sinc expansion and to the DE formula. $d^{\prime}$ is the half-width of the strip domain

$$
|\operatorname{Im} t|<d^{\prime}
$$

on which $f(\psi(t)) \psi^{\prime}(t)$ is analytic. $E_{\text {int }}$ is the error due to numerical integration, while $E_{\text {trunc }}$ is the error due to truncation of the summation.

First, for $f(x)$ we think of $y\left(\mu_{m} S_{k} \rho\right)^{(m)}, m=0,1,2$ and $\sigma S_{k} \rho$. For each term $d^{\prime}$ is different with each other. Note that, if $d \approx d^{\prime}$ holds the error $E_{\text {int }}$ in (4.9) due to the DE formula for numerical integration is about as squared small as the one due to DE-sinc expansion (3.1) with the same mesh size $h$. And hence, we assume here that

$$
2 d^{\prime} \geq d
$$

and, under this assumption, we can ignore $E_{\text {int }}$ compared with $E_{\text {sinc }}(h)$ in $(3.1)$.

Next, note that each term of sum (3.1) has a factor $1 / \phi^{\prime}\left(x_{j}\right)$ which decays double exponentially as seen from (4.3) and, consequently, the sum (4.9) is truncated so that the absolute value of the integrand is less than $\varepsilon_{\mathrm{tr}}$. In view of these behavior we see that the error $E_{\text {trunc }}$ in (4.9) due to truncation can be ignored in actual computation.

Now we are ready to write an explicit form of the equation which we solve. If we apply the DE formula (4.9) to each term in (4.8) and truncate the infinite sum 
according to the strategy discribed in Section 3, we eventually obtain the following system of linear algebraic equations:

$$
\begin{aligned}
& \sum_{j=-n_{-}}^{n_{+}}\left\{\mu_{2} \delta_{j k}^{(2)}+h\left(-\mu_{2}\left(\frac{1}{\phi^{\prime}}\right)^{\prime}+\mu_{1}\left(\frac{1}{\phi^{\prime}}\right)\right)\left(x_{j}\right) \delta_{j k}^{(1)}\right. \\
& \left.\quad+h^{2}\left(\mu_{2}\left(\frac{1}{\phi^{\prime}}\right)^{\prime \prime}\left(\frac{1}{\phi^{\prime}}\right)-\mu_{1}^{\prime}\left(\frac{1}{\phi^{\prime}}\right)^{2}-\mu_{1}\left(\frac{1}{\phi^{\prime}}\right)^{\prime}\left(\frac{1}{\phi^{\prime}}\right)+\mu_{0}\left(\frac{1}{\phi^{\prime}}\right)^{2}\right)\left(x_{j}\right) \delta_{j k}^{(0)}\right\} y_{j} \\
& =h^{2} \sigma\left(\frac{1}{\phi^{\prime}}\right)^{2}\left(x_{k}\right), \quad k=-n_{-},-n_{-}+1, \ldots, n_{+},
\end{aligned}
$$

where

$$
\delta_{j k}^{(0)}=\left\{\begin{array}{ll}
1 ; & j=k, \\
0 ; & j \neq k,
\end{array} \quad \delta_{j k}^{(1)}=\left\{\begin{array}{ll}
0 ; & j=k, \\
\frac{(-1)^{k-j}}{(k-j)} ; & j \neq k,
\end{array} \quad \delta_{j k}^{(2)}= \begin{cases}-\frac{\pi^{2}}{3} ; & j=k, \\
\frac{-2(-1)^{k-j}}{(k-j)^{2}} ; & j \neq k .\end{cases}\right.\right.
$$

Explicit form of each derivative term which appears in (4.12) can be written as follows:

$$
\begin{aligned}
& \left(\frac{1}{\phi^{\prime}}\right)(x)=\left(\frac{1}{\phi^{\prime}}\right)(\psi(t))=\frac{b-a}{2} \frac{\frac{\pi}{2} \cosh t}{\cosh ^{2}\left(\frac{\pi}{2} \sinh t\right)}, \\
& \left(\frac{1}{\phi^{\prime}}\right)^{\prime}=\frac{d}{d x}\left(\frac{1}{\phi^{\prime}}\right)=\psi^{\prime}(x) \frac{d}{d t}\left(\frac{1}{\phi^{\prime}}\right)=\tanh t-\pi \cosh t \tanh \left(\frac{\pi}{2} \sinh t\right) \\
& \left(\frac{1}{\phi^{\prime}}\right)^{\prime \prime}\left(\frac{1}{\phi^{\prime}}\right)=\frac{1}{\cosh ^{2} t}-\pi \sinh t \tanh \left(\frac{\pi}{2} \sinh t\right)-\frac{\pi^{2}}{2} \frac{\cosh ^{2} t}{\cosh ^{2}\left(\frac{\pi}{2} \sinh t\right)} .
\end{aligned}
$$

We summarize here the procedure for computing numerical solution

$$
\tilde{y}_{n_{\mathrm{tot}}}(x)=\sum_{j=-n_{-}}^{n_{+}} y_{j} S(j, h)\left(\psi^{-1}(x)\right)
$$

of the boundary value problem of second order ordinary differential equation (4.1) including singularly perturbed problem (1.1). $n_{\text {tot }}$ which appears in the subscript of the left hand side of (4.17) is defined by

$$
n_{\mathrm{tot}}=n_{-}+n_{+}+1
$$

and denotes the total number of terms in (4.17) actually computed in (4.12).

1. Select a small number $\varepsilon_{\mathrm{tr}}$ for truncation (machine epsilon, for example).

2. Select a mesh size $h$.

3. Determine $n_{-}$and $n_{+}$using (3.10) and (3.11), or using (3.15) and (3.16), repectively. 
4. Solve system of linear algebraic equations (4.12) with $\mu_{2}(x)=\varepsilon$ for $y_{j}, j=$ $-n_{-},-n_{-}+1, \ldots, n_{+} \cdot y_{j}$ is the value of approximate solution at the sinc point $x=\psi(j h)$, i.e., $y_{j}=\tilde{y}_{n_{\text {tot }}}\left(x_{j}\right)$.

5. The value of approximate solution at an arbitrary $x$ is obtained by computing the right hand side of (4.17).

Let $E_{\max }$ be the error of approximate solution. If we plot a graph of $E_{\max }$ in $\operatorname{logarithmic}$ scale, i.e., $\log E_{\max }$ vs. $n_{\text {tot }}$, we find an approximately linear relation $\log E_{\max } \approx-c n_{\text {tot }}$. The reason is as follows. From (3.10) and (3.11), or from (3.15) and (3.16), we see that

$$
h \approx t_{\mathrm{tot}} / n_{\mathrm{tot}}, \quad t_{\mathrm{tot}}=t_{-}+t_{+}
$$

holds, so that from (3.1) we have

$$
E_{\max } \approx\left|E_{\text {sinc }}(h)\right|=O\left(\exp \left(-\frac{\pi d}{h}\right)\right)=O\left(\exp \left(-c n_{\text {tot }}\right)\right), \quad c=\frac{\pi d}{t_{\text {tot }}} .
$$

Note, however, that this relation holds only for such $h$ or $n_{\text {tot }}$ that satisfies $\left|E_{\text {sinc }}(h)\right| \geq \varepsilon_{\text {tr }}$.

\section{Numerical solution of semi-linear problem}

The method described in the preceding sections can be applied to numerical solution of semi-linear boundary value problems with homogeneous boundary condition of the following form including singularly perturbed problem with $\mu_{2}(x)=\varepsilon$ :

$$
\begin{aligned}
& \mu_{2}(x) y^{\prime \prime}(x)+\mu_{1}(x) y^{\prime}(x)+F[x, y]=\sigma(x), \quad a<x<b, \\
& y(a)=y(b)=0 .
\end{aligned}
$$

$F[x, y]$ is a non-linear term and we assume that $F[x, y]$ does not include derivatives of $y(x)$. We also assume that $F[x, y]$ is a function of $x$ and $y$ analytic on $a \leq x \leq b$. We again assume solution of the form (4.17) and repeat the same analysis. Then we finally obtain the following system of nonlinear algebraic equations:

$$
\begin{aligned}
\sum_{j=-n_{-}}^{n_{+}}[ & \left\{\mu_{2} \delta_{j k}^{(2)}+h\left(-\mu_{2}\left(\frac{1}{\phi^{\prime}}\right)^{\prime}+\mu_{1}\left(\frac{1}{\phi^{\prime}}\right)\right)\left(x_{j}\right) \delta_{j k}^{(1)}\right. \\
& \left.+h^{2}\left(\mu_{2}\left(\frac{1}{\phi^{\prime}}\right)^{\prime \prime}\left(\frac{1}{\phi^{\prime}}\right)-\mu_{1}^{\prime}\left(\frac{1}{\phi^{\prime}}\right)^{2}-\mu_{1}\left(\frac{1}{\phi^{\prime}}\right)^{\prime}\left(\frac{1}{\phi^{\prime}}\right)\right)\left(x_{j}\right) \delta_{j k}^{(0)}\right\} y_{j} \\
& \left.+h^{2} F\left[x_{j}, y_{j}\right]\left(\frac{1}{\phi^{\prime}}\right)^{2}\left(x_{j}\right) \delta_{j k}^{(0)}\right]=h^{2} \sigma\left(\frac{1}{\phi^{\prime}}\right)^{2}\left(x_{k}\right), \quad k=-n_{-},-n_{-}+1, \ldots, n_{+} .
\end{aligned}
$$

If we solve (5.2) for $y_{j}, j=-n_{-},-n_{-}+1, \ldots, n_{+}$we get approximate solution $y_{j}=\tilde{y}_{n_{\mathrm{tot}}}\left(x_{j}\right)$ at the sinc point $x_{j}=\psi(j h)$, and from (4.17) we obtain approximate solution at an arbitrary $x$. If we start from a good initial guess we usually can obtain a solution of (5.2) using Newton's method. In Section 7 we give an example of numerical solution of a semi-linear problem. 


\section{DE sinc-collocation method}

We can derive a sinc-collocation method based on the DE transformation, which we call the DE-sinc collocation method, for solving the boundary value problem of second order ordinary differential equation (4.1) with homogeneous boundary condition, singularly perturbed problem when $\mu_{2}(x)=\varepsilon$, in a similar way as in the case of the sinc-Galerkin method. In (4.1) we replace $y(x)$ with $\tilde{y}_{h}(x)$ given by (3.2), multiply $h^{2} /\left\{\phi^{\prime}(x)\right\}^{2}$ on both sides and substitute $x$ with $x_{k}=\psi(k h)$. Then we obtain an approximate equation based on the collocation:

$$
h^{2} \mu_{2} \tilde{y}_{h}^{\prime \prime}\left(\frac{1}{\phi^{\prime}}\right)^{2}\left(x_{k}\right)+h^{2} \mu_{1} \tilde{y}_{h}^{\prime}\left(\frac{1}{\phi^{\prime}}\right)^{2}\left(x_{k}\right)+h^{2} \mu_{0} \tilde{y}_{h}\left(\frac{1}{\phi^{\prime}}\right)^{2}\left(x_{k}\right)=h^{2} \sigma\left(\frac{1}{\phi^{\prime}}\right)^{2}\left(x_{k}\right) \text {. }
$$

In equation (6.1) we actually differentiate $S(j, h)\left(\psi^{-1}(x)\right)$ in $\tilde{y}_{h}^{\prime}(x)$ and $\tilde{y}_{h}^{\prime \prime}(x)$ of (3.2) with respect to $x$ and then substitute $x$ with $x_{k}=\psi(k h)$. When we carry out differentiation we can use the formulas (4.14), (4.15) and (4.16). Finally we obtain

$$
\begin{aligned}
& \sum_{j=-n_{-}}^{n_{+}}\left\{\mu_{2}\left(x_{k}\right) \delta_{j k}^{(2)}+h\left(-\mu_{2}\left(\frac{1}{\phi^{\prime}}\right)^{\prime}+\mu_{1}\left(\frac{1}{\phi^{\prime}}\right)\right)\left(x_{k}\right) \delta_{j k}^{(1)}+h^{2} \mu_{0}\left(\frac{1}{\phi^{\prime}}\right)^{2}\left(x_{k}\right) \delta_{j k}^{(0)}\right\} y_{j} \\
& =h^{2} \sigma\left(\frac{1}{\phi^{\prime}}\right)^{2}\left(x_{k}\right), \quad k=-n_{-},-n_{-}, \ldots, n_{+}
\end{aligned}
$$

If we solve this system of linear algebraic equations for $y_{j}, j=-n_{-}, \ldots, n_{+}$we get approximate solution $y_{j}=\tilde{y}_{n_{\text {tot }}}\left(x_{j}\right)$ at the sinc point $x_{j}=\psi(j h)$, and from (4.17) we obtain approximate solution at an arbitrary $x$.

\section{Numerical examples}

In this section we present numerical examples for singularly perturbed boundary value problems (1.1) and (5.1) with $\mu_{2}(x)=\varepsilon$ to illustrate the result of analysis discussed in the preceding sections. In order to highlight high accuracy in the present method solution of the problems was computed in quadruple precision arithmetic using Fujitsu Fortran compiler on a Pentium IV personal computer. Machine epsilon of this system is $1.926 \times 10^{-34}$ and we selected $\varepsilon_{\mathrm{tr}}=1.926 \times 10^{-34}$ for truncation parameter used in (3.8). When we carry out numerical computation $\varepsilon_{\text {tr }}$ is computed first in the computer [3] and then is used in the subsequent computation.

In all the examples the problem is defined on $0<x<1$, so that we set $a=0$ and $b=1$, and hence the DE transformation is

$$
x=\psi(t)=\frac{1}{2} \tanh \left(\frac{\pi}{2} \sinh t\right)+\frac{1}{2}=\frac{1}{2} \frac{\exp \left(\frac{\pi}{2} \sinh t\right)}{\cosh \left(\frac{\pi}{2} \sinh t\right)}=\frac{1}{1+\exp (-\pi \sinh t)} .
$$

In every example we selected $h=0.32,0.16,0.08,0.04,0.02,0.01$, and supplemented other suitable values of $h$ if it makes the error behavior clearer. Then we carried out computation following the procedure described at the end of Section 4 . 
For each example we show the maximum of the error of numerical solution $y_{j}=\tilde{y}_{n_{\text {tot }}}\left(x_{j}\right)$ at the sinc points $x_{j}=\psi(j h), j=-n_{-},-n_{-}+1, \ldots, n_{+}$, i.e.,

$$
E_{\max }=\max _{-n_{-} \leq j \leq n_{+}}\left|y_{j}-y\left(x_{j}\right)\right|, \quad x_{j}=\psi(j h)
$$

since we know the exact solution $y(x)$ in every problem. In each figure 'max error' denotes $E_{\max }$ and ' $n_{\text {tot }}$ ' denotes the total number of terms $n_{\text {tot }}=n_{-}+n_{+}+1$ in the sinc expansion. Each marker on the curves in the figure corresponds to a data pair $\left(n_{\text {tot }}, E_{\max }\right)$ which we obtained in actual computation, and for each $\varepsilon$ we connected adjacent data pairs with a line segment.

Example 1. The first example is a problem of standard type of singular perturbation from $[1,2]$ :

$$
\begin{aligned}
& \varepsilon y^{\prime \prime}(x)-y(x)=\cos ^{2}(\pi x)+2 \varepsilon \pi^{2} \cos (2 \pi x), \quad 0<x<1, \\
& y(0)=y(1)=0 .
\end{aligned}
$$

This equation corresponds to the case $a=0, b=1, \mu_{1}(x)=0$ and $\mu_{0}(x)=-1$ in (1.1), and hence solution $y(x)$ satisfies (3.12) with $\alpha=1$ and we select $L_{-}=1 / \sqrt{\varepsilon}$ as well as $L_{+}=1 / \sqrt{\varepsilon}$. Actually the exact solution of this problem is

$$
y(x)=\frac{e^{-x / \sqrt{\varepsilon}}+e^{-(1-x) / \sqrt{\varepsilon}}}{1+e^{-1 / \sqrt{\varepsilon}}}-\cos ^{2}(\pi x) .
$$

We set $\beta_{-}=\beta_{+}=1$ and solved this problem using the DE sinc-Galerkin method and error $E_{\max }$ of numerical solution with $\varepsilon=10^{-10}, 10^{-8}, 10^{-5}$ and 1 is shown in Fig. 1. In Table 1 we show a list of $t_{-}$and $t_{+}$defined in (3.15) and (3.16). In the present example $t_{-}=t_{+}$holds for each $\varepsilon$. From Table 1 and from (4.19) we can easily get the value of $h$ corresponding to $n_{\text {tot }}$. We see that we obtained a result with almost as high accuracy as quadruple precision using sinc expansion with a few hundred terms. In every case of $\varepsilon=10^{-10}, 10^{-8}$ and $10^{-5}$ we did not pay any particular care for smallness of $\varepsilon$ as in the case $\varepsilon=1$ except selection of values of $L_{-}$and $L_{+}$.

Table 1. $t_{-}$and $t_{+}$for each $\varepsilon$ in Example 1. $t_{-}=t_{+}$in this example.

\begin{tabular}{|c|c|c|c|c|}
\hline$\varepsilon$ & $10^{-10}$ & $10^{-8}$ & $10^{-5}$ & 1 \\
\hline$t_{-}, t_{+}$ & 4.0387 & 4.0125 & 3.9719 & 3.9004 \\
\hline
\end{tabular}

Incidentally, if we use $L_{-}=L_{+}=1$ instead of $L_{-}=L_{+}=1 / \sqrt{\varepsilon}$ in Example 1 , we will get almost the same curve as in Fig. 1 for each $\varepsilon$. The reason is that effect of $L_{-}$and $L_{+}$on numerical solution is not large in general as is mentioned at the end of Section 3. See also Example 3. 


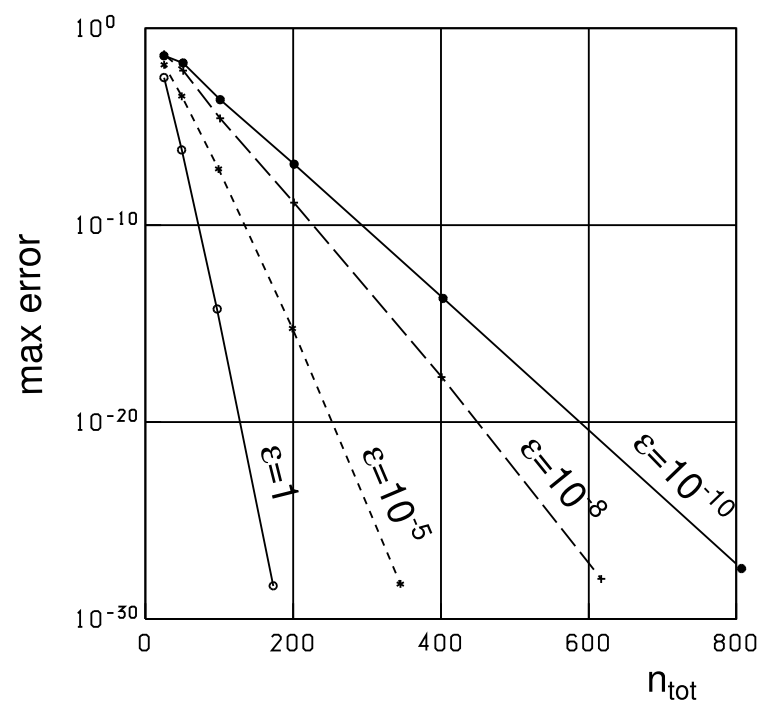

Fig. 1. Maximum error $E_{\max }$ in Example 1 with $\varepsilon=10^{-10}, 10^{-8}, 10^{-5}$, and 1 .

In Fig. 1 we observe that the error presents almost linear dependence, i.e., $\log E_{\max } \approx-c n_{\text {tot }}$, or $E_{\max }=O\left(\exp \left(-c n_{\text {tot }}\right)\right)$ as is mentioned at the end of Section 4. If, however, you make $h$ smaller in each case of $\varepsilon$ than plotted in Fig. 1 you will find that the result will stray off the linear dependence due to accumulation of round-off error.

It is natural to set $\varepsilon_{\mathrm{tr}}=1.926 \times 10^{-34}$ if a solution with accuracy of about quadruple precision is required. However, if, for example, a solution with accuracy of double precision is required selection of $\varepsilon_{\mathrm{tr}}=1.926 \times 10^{-34}$ seems to be too severe. In fact this selection results in redundant evaluations of terms in the sinc expansion. In Fig. 2 we show comparison in the case $\varepsilon=10^{-10}$ between the solution with $\varepsilon_{\mathrm{tr}}=1.926 \times 10^{-34}$ and the one with $\varepsilon_{\mathrm{tr}}=2.220 \times 10^{-16}$ which is equal to machine epsilon in double precision arithmetic of the system we used. Solid curve represents error with $\varepsilon_{\mathrm{tr}}=1.926 \times 10^{-34}$, while the dashed curve represents the one with $\varepsilon_{\mathrm{tr}}=2.220 \times 10^{-16}$. Although computation was carried out in quadruple precision arithmetic also in the case $\varepsilon_{\mathrm{tr}}=2.220 \times 10^{-16}$ the error curve with dashed line does not change so much if computation is carried out in double precision arithmetic as long as we select $\varepsilon_{\mathrm{tr}}=2.220 \times 10^{-16}$. We see that actually we need a smaller number of terms when we require a result with lower accuracy, although the differnece is not so large, as seen in Fig. 2. Incidentally, in this comparison $t_{-}\left(=t_{+}\right)=4.0387$ when $\varepsilon_{\mathrm{tr}}=1.926 \times 10^{-34}$, while $t_{-}\left(=t_{+}\right)=3.4103$ when $\varepsilon_{\mathrm{tr}}=$ $2.220 \times 10^{-16}$.

Since criterion (3.8) for truncation with $\varepsilon_{\mathrm{tr}}=$ machine epsilon is severe in general there remain redundant terms without being discarded by truncation. On the other hand, the double exponential decay not only is steep but also comes on a sudden, so that the number of these redundant terms is usually not large. See p. 440 in [7] for detailed discussion. 


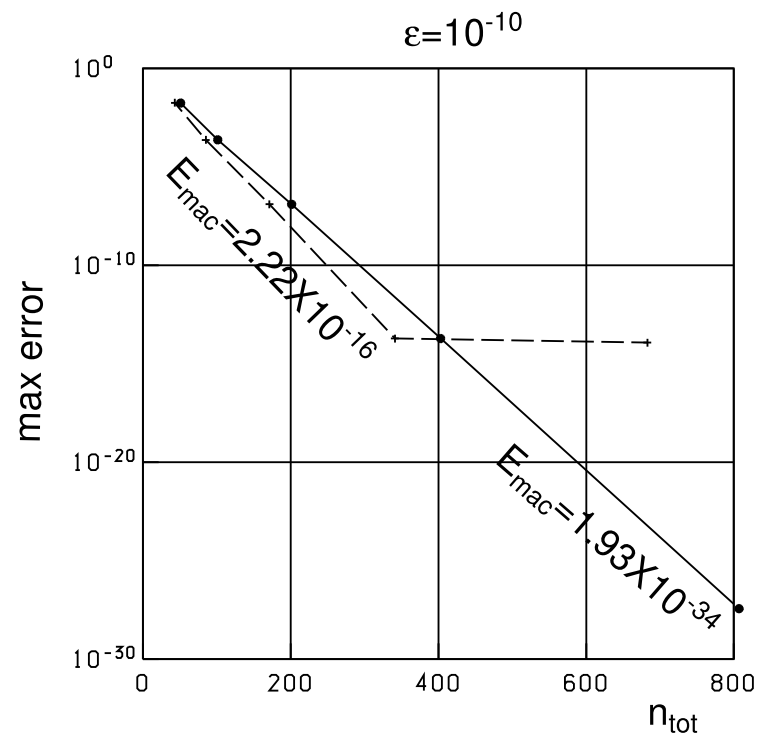

Fig. 2. Comparison between $\varepsilon_{\operatorname{tr}}=1.926 \times 10^{-34}$ and $\varepsilon_{\operatorname{tr}}=2.220 \times 10^{-16}$ when $\varepsilon=10^{-10}$ in Example 1.

In order to see how smooth the sinc expansion looks we divided the interval $0 \leq x \leq 1$ into 1000 equal subintervals with mesh size 0.001 and computed approximate solution $\tilde{y}_{n_{\text {tot }}}\left(x_{i}\right)$ from (4.17) with $h=0.08$ at $x_{i}=i / 1000,1 \leq i \leq 999$ for the case $\varepsilon=10^{-5}$, and plotted the numerical solution as a graph in Fig. 3. This approximate solution is so close to the exact solution that if we overplot the exact solution on the graph we cannot discriminate these two graphs. The maximum error $E_{\max }=7.04 \times 10^{-8}$ on the sinc points is attained at $j=-14$, i.e., $x=1.34 \times 10^{-2}$, while the maximum error $8.59 \times 10^{-7}$ on the equi-distributed mesh is attained at $i=7$, i.e., $x=7.00 \times 10^{-3}$. In this figure we find boundary layers at both ends of the interval.

We also plotted location of the sinc points at the bottom of Fig. 3. We find that sampling points based on the DE transformation accumulate to the boundary edge.

We computed numerical solution of the same problem (7.3) with $\varepsilon=10^{-5}$ by the DE sinc-collocation method described in Section 6. Selection of $h, t_{-}$and $t_{+}$, so that determination of $n_{-}$and $n_{+}$, are the same as in the sinc-Galerkin method based on the DE transformation. In Fig. 4 the error $E_{\max }$ (DE-col) is shown together with the error by the sinc-Galerkin method (DE-Gal). Error curve with the DE sinc-collocation method differes a little from that with the DE sincGalerkin method. We see that, if we compare errors at the same $n_{\text {tot }}$, error by the DE sinc-Galerkin method is slightly smaller than error by the sinc-collocation method. This tendency is observed also in other examples. However, it should be mentioned that the DE sinc-collocation method has a merit that the coefficient of equation (6.2) is less complicated than that of (4.12). 


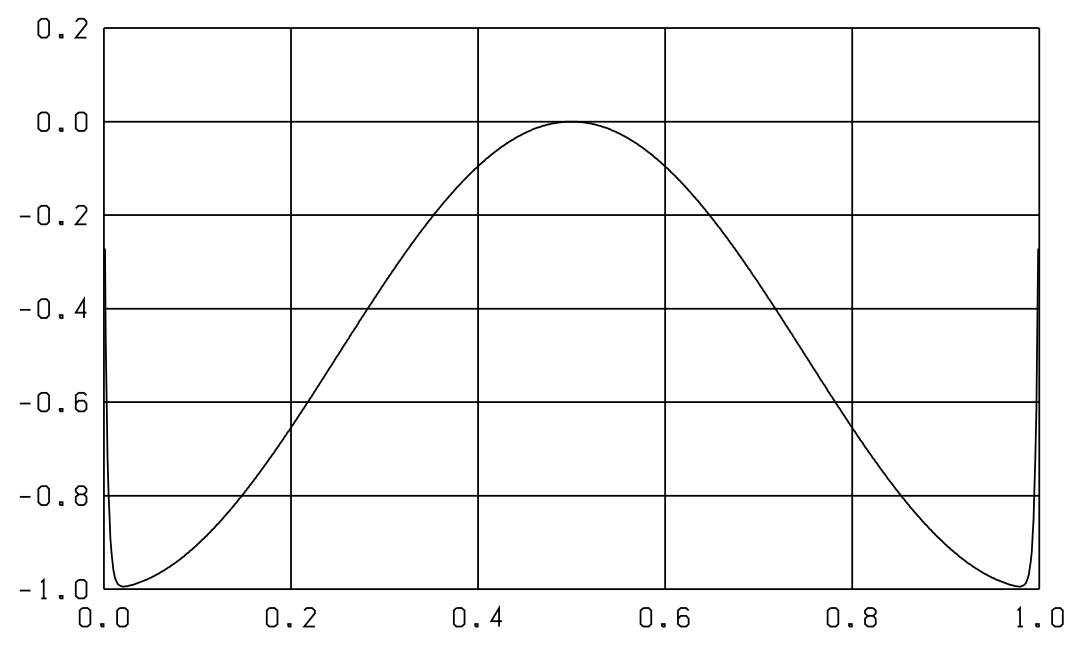

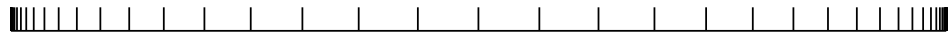

Fig. 3. Numerical solution of (7.3) by the DE sinc-Galerkin method for $\varepsilon=10^{-5}$ with $h=0.08$.

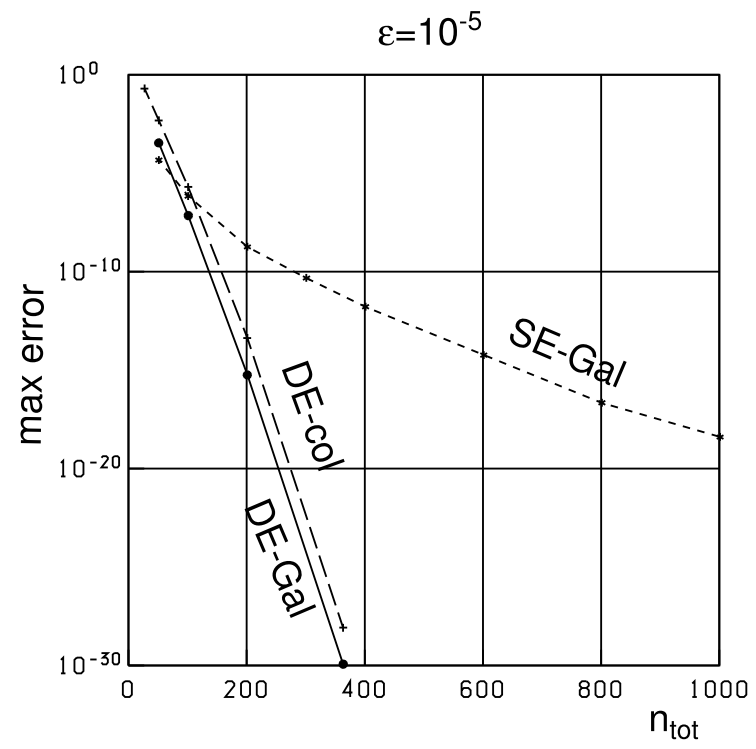

Fig. 4. Comparison between the DE transformation and the SE transformation when $\varepsilon=10^{-5}$ in Example 1 .

We solved the problem also using the SE sinc-Galerkin method. In this case we set $d=\pi / 2, n=100$ and hence $h=\pi / \sqrt{n}$ in accordance with [2] and followed the conventional procedure of the SE sinc-Galerkin method. The result is shown also in Fig. 4 (SE-Gal). We see that convergence by the DE sinc-Galerkin method or by the DE sinc-collocation method is much faster than convergence by the SE sinc-Galerkin method as is expected from (3.6) and (3.7). 
Example 2. Next example is a problem from Liu and $\mathrm{Xu}[5]$ as one of the recent results using a conventional method:

$$
\begin{aligned}
& \varepsilon y^{\prime \prime}(x)-(2+\sin x) y(x)=f(x), \quad 0<x<1, \\
& y(0)=y(1)=0 .
\end{aligned}
$$

$f(x)$ is chosen so that

$$
y(x)=\exp (-x / \sqrt{\varepsilon})+\exp (-(1-x) / \sqrt{\varepsilon})+x(1-x)-(1+\exp (-1 / \sqrt{\varepsilon}))
$$

is the exact solution. This equation corresponds to $a=0, b=1, \mu_{1}(x)=0$ and $\mu_{0}(x)=-(2+\sin x)$ in (1.1), so that it satisfies (3.12) with $\alpha=1$. And hence we select $L_{-}=1 / \sqrt{\varepsilon}$ and $L_{+}=1 / \sqrt{\varepsilon}$. Liu and Xu solved this singularly perturbed problem by the Galerkin method based on Hermite splines with knots, or sampling points, adapted to the singular behavior of the solution. On the other hand we solved the same problem using the DE sinc-Galerkin method whose sampling points are defined based on the DE transformation. We set $\beta_{-}=\beta_{+}=1$. For the same reason as in Example 1 we used $L_{-}=1 / \sqrt{\varepsilon}$ and $L_{+}=1 / \sqrt{\varepsilon}$.

In Fig. 5 error by our method (DE-Gal) together with error by Liu and $\mathrm{Xu}$ directly copied from Table 3 in [5] (Hermite splines) is shown for the case $\varepsilon=$ $1.456 \times 10^{-11}\left(\sqrt{\varepsilon}=3.816 \times 10^{-6}\right) . \quad n_{\text {tot }}$ denotes total number of the sampling points, and $t_{-}\left(=t_{+}\right)$is 4.0494 in the present case. In Fig. 5 we find that the Galerkin method based on Hermite splines with adapted knots by Liu and Xu gives better result for small $n_{\text {tot }}$. However, as $n_{\text {tot }}$ increases the present method beats it and eventually presents much faster convergence.

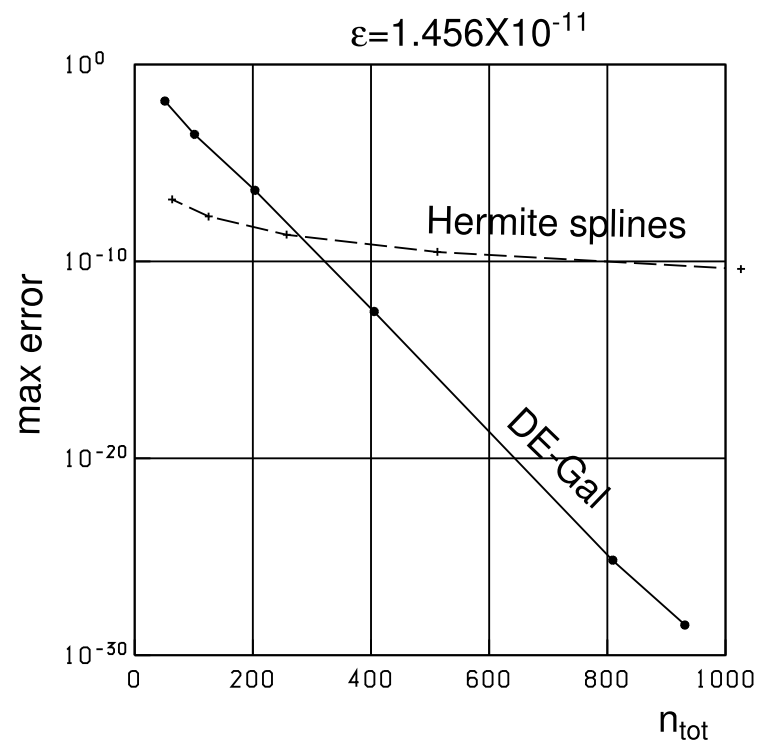

Fig. 5. Comparison between the DE sinc-Galerkin method and the Galerkin method based on the Hermite splines with adapted knots in Example 2. 
Example 3. Next example is a semi-linear problem again from [1, 2]:

$$
\begin{aligned}
& \varepsilon y^{\prime \prime}(x)+2 y^{\prime}(x)+y^{2}(x)=(\exp (-x / \varepsilon)-1 / \varepsilon) \exp (-x / \varepsilon), \quad 0<x<1, \\
& y(0)=1, \quad y(1)=\exp (-1 / \varepsilon) .
\end{aligned}
$$

The exact solution is

$$
y(x)=\exp (-x / \varepsilon)
$$

This problem is not only of semi-linear equation but also has inhomogeneous boundary condition. In order to use the DE-sinc method we need to modify the problem into a problem with homogeneous boundary condition. In the present problem we define a linear function

$$
s(x)=(\exp (-1 / \varepsilon)-1) x+1
$$

which satisfies inhomogeneous boundary condition and write

$$
u(x)=y(x)-s(x) .
$$

After this modification we see that $u(x)$ satisfies

$$
\begin{aligned}
& \varepsilon u^{\prime \prime}(x)+2 u^{\prime}(x)+u^{2}(x)+2((\exp (-1 / \varepsilon)-1) x+1) u(x)=f(x), \\
& u(0)=u(1)=0,
\end{aligned}
$$

where $f(x)$ is chosen so that

$$
u(x)=\exp (-x / \varepsilon)-(\exp (-1 / \varepsilon)-1) x-1
$$

is the exact solution. We set $\beta_{-}=\beta_{+}=1$ and solved equation (7.11) numerically for $u(x)$ and substitued $u(x)$ in (7.10) with this numerical solution to obtain $\tilde{y}_{n_{\text {tot }}}(x)$ in $(4.17)$.

We can see from [12] that if $\mu_{1}$ and $F[x, u]$ satisfy

$$
0<\alpha \leq \mu_{1}(x)(\alpha>0), \quad 0 \leq F_{u} \leq \beta(x)
$$

in (5.1) with $\mu_{2}(x)=\varepsilon$, solution $u(x)$ satisfying homogeneous boundary condition $u(0)=0$ has a boundary layer only at the left edge point $x=0$ and includes a component $u_{-}(x)$ which dominates in the boundary layer with a bound $\hat{u}_{-}(x)$ such that

$$
|u(x)| \approx\left|u_{-}(x)\right| \leq \hat{u}_{-}(x), \quad \hat{u}_{-}(x)=C_{-}\left|\exp \left(-\alpha \frac{x}{\varepsilon}\right)-1\right| .
$$

The right edge point is a regular zero. 
The present equation (7.11) corresponds to $a=0, b=1, \mu_{2}(x)=\varepsilon, \mu_{1}(x)=2$ and $F[x, u]=u^{2}+2((\exp (-1 / \varepsilon)-1) x+1) u$ in $(5.1)$, so that it satisfies (7.13) with $\alpha=2$ (since we know the exact solution (7.12)), and hence we select $L_{-}=2 / \varepsilon$, $L_{+}=1$. In this example we computed numerical solution also with selection $L_{-}=1$, $L_{+}=1$. In the latter selection we do not take into consideration smallness of $\varepsilon$ at all except that we just set $\mu_{2}(x)=\varepsilon$ in (5.2) as a standard boundary value problem. In Table 2 we show $t_{-}$and $t_{+}$used in numerical solution in the case $L_{-}=\alpha / \varepsilon$ $(\alpha=2), L_{+}=1$. For $L_{-}=1, L_{+}=1$ we have $t_{-}=t_{+}=3.9004$.

Table 2. $t_{-}$and $t_{+}$for the non-linear problem in Example 3.

\begin{tabular}{|c|c|c|}
\hline$\varepsilon$ & $10^{-10}$ & $10^{-5}$ \\
\hline$t_{-}$ & 4.1670 & 4.0464 \\
\hline$t_{+}$ & 3.9004 & 3.9004 \\
\hline
\end{tabular}

In order to solve non-linear system of algebraic equations (5.2) we employed Newton's method starting from an initial guess $u^{(0)}(x) \equiv 0$ which is a linear function satisfying homogeneous boundary condition. In every case of $\varepsilon$ Newton's method converged at most with 5 iterations.

In Fig. 6 we show the error $E_{\max }$ of numerical solution of the modified problem (7.11) for $\varepsilon=10^{-10}$ and $10^{-5}$. Solid curves correspond to the selection $L_{-}=1$, $L_{+}=1$, while dotted curves correspond to the selection $L_{-}=2 / \varepsilon, L_{+}=1$. From Fig. 6 we see that, although accuracy in the selection $L_{-}=1, L_{+}=1$ is slightly higher than that in the selection $L_{-}=2 / \varepsilon, L_{+}=1$, difference between these two selections is quite small as is discussed at the end of Section 3.

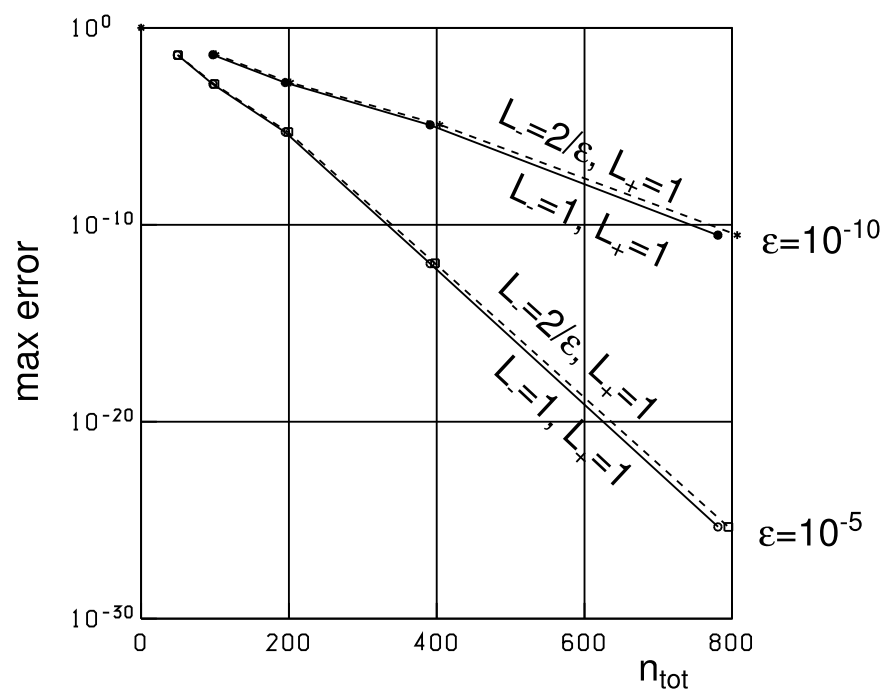

Fig. 6. Error of nonlinear problem (7.11). 
Example 4. The last example is a problem which has both a boundary layer and an algebraic singularity at $x=0$ and $x=1$.

$$
\begin{aligned}
& \varepsilon y^{\prime \prime}(x)-y(x)=1+\varepsilon(\sqrt{x(1-x)})^{\prime \prime}-\sqrt{x(1-x)}, \quad 0<x<1, \\
& y(0)=y(1)=0 .
\end{aligned}
$$

The exact solution is

$$
y(x)=\frac{\exp (-x / \sqrt{\varepsilon})+\exp (-(1-x) / \sqrt{\varepsilon})}{1+\exp (-1 / \sqrt{\varepsilon})}-1+\sqrt{x(1-x)} .
$$

We set $\beta_{-}=\beta_{+}=1 / 2$. We selected $L_{-}=L_{+}=1$ and solved this problem using the present method. In Fig. 7 the error of the result for $\varepsilon=10^{-6}, 10^{-3}$, 1 is shown. From this figure we find that the present method works quite well also for a singularly perturbed problem with an algebraic singularity at the end point.

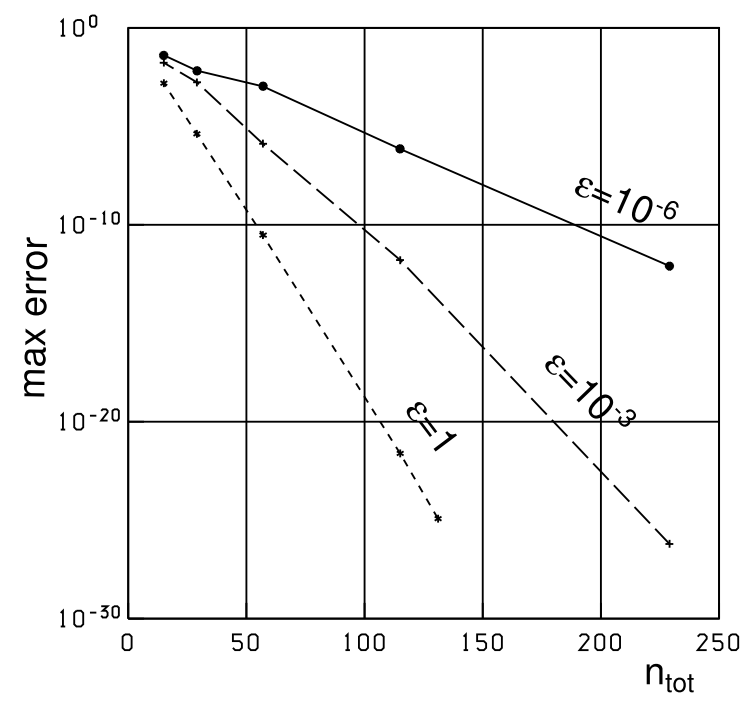

Fig. 7. Error of problem (7.15) with end-point algebraic singularity.

\section{Estimation of the half width $d$ of the strip domain in which $y(\psi(t))$ is analytic}

As is already mentioned it is usually difficult to get the value of $d$, the half width of the domain $|\operatorname{Im} t|<d$ on which $y(\psi(t))$ is analytic, before we start numerical computation. On the other hand, after computation, we can estimate the value of $d$ from dependence of the error $E_{\max }$ on $1 / h$. If we plot $-\log \left(E_{\max }\right)$ vs. $1 / h$ curve almost linear dependence is observed as is expected from $E_{\text {sinc }}(h)=O(\exp (-\pi d / h))$ in (3.1). In Fig. 8 we show error curves of numerical solution in Example 1 as a function of $1 / h$. Computation was carried out with the same selection of $L_{-}$and $L_{+}$as that in Example 1, i.e., $L_{-}=L_{+}=1 / \sqrt{\varepsilon}$. In fact, dependence of the error 
in logarithmic scale on $1 / h$ looks almost linear. Since gradient of each curve is expected to give $\pi d$ for each $\varepsilon$ from (3.1) we applied linear curve fitting. We assumed that $-\log \left(E_{\max }\right)=A \times(1 / h)+B$ holds and calculated $A$ and $B$ by the least squares method with equal weight for all the data. We used such pairs of data $\left(1 / h,-\log \left(E_{\max }\right)\right)$ that seem to lie consecutively almost on the linear part of each curve as is plotted in Fig. 8. Values of $A, B$, and calculated $\tilde{d}$, i.e., $A / \pi$, are shown in Table 3. From this table we see that value of $\tilde{d}$ is considerably different from $\pi / 2$. Theoretical analysis for pre-estimation of $d$ is left to the future work.

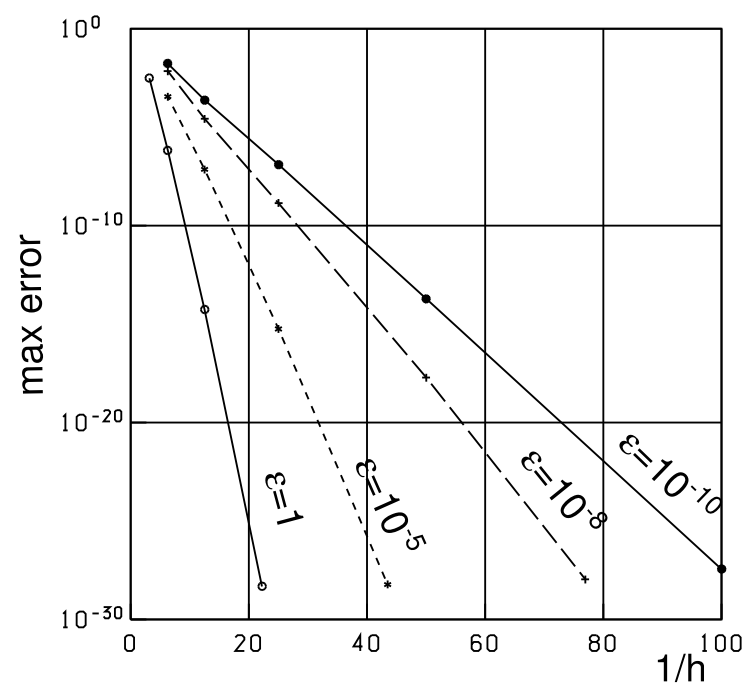

Fig. 8. Error of problem (7.3) as a function of $1 / h$.

Table 3. Dependence of $-\log \left(E_{\max }\right)$ on $1 / h$.

\begin{tabular}{|l|c|r|c|}
\hline \multicolumn{1}{|c|}{$\varepsilon$} & $A$ & \multicolumn{1}{c|}{$B$} & $\tilde{d}$ \\
\hline $10^{-10}$ & 0.629 & 0.258 & 0.200 \\
\hline $10^{-8}$ & 0.836 & -0.293 & 0.266 \\
\hline $10^{-5}$ & 1.537 & -2.394 & 0.489 \\
\hline 1 & 3.126 & -4.943 & 0.995 \\
\hline
\end{tabular}




\section{References}

[ 1 ] E.P. Doolan, J.J.H. Miller and W.H.A. Schilders, Uniform Numerical Methods for Problems with Initial and Boundary Layers. Boole Press, Dublin, Ireland, 1980.

[2] M. El-Gamel and J.R. Cannon, On the solution a of second order singularly perturbed boundary value problem by the sinc-Galerkin method. Z. angew. Math. Phys., 56 (2005), 45-58.

[ 3 ] G.E. Forsythe, M.A. Malcolm and C.B. Moler, Computer Method for Mathematical Computations. Prentice-Hall, 1977.

[ 4 ] M.K. Kadalbajoo and Y.N. Reddy, An initial-value technique for a class of nonlinear singular perturbation problems. J. Opt. Theo. Appl., 53 (1987), 395-406.

[ 5 ] S.-T. Liu and Y. Xu, Galerkin methods based on Hermite splines for singular perturbation problems. SIAM J. Numer. Anal., 43 (2006), 2607-2623.

[ 6 ] J. Lund and K.L. Bowers, Sinc Methods for Quadrature and Differential Equations. SIAM, Philadelphia, 1992.

[ 7 ] M. Muhammad and M. Mori, Double exponential formulas for numerical indefinite integration. J. Comput. Appl. Math., 161 (2003), 431-448.

[ 8] M. Mori and M. Sugihara, The double exponential transformation in numerical analysis. Numerical Analysis in the 20th Century, Vol. V: W. Gautschi, F. Marcellán and L. Reichal (eds.), Quadrature and Orthogonal Polynomials, J. Comput. Appl. Math., 127 (2001), 287-296.

[ 9 ] A. Nurmuhammad, M. Muhammad, M. Mori and M. Sugihara, Double exponential transformation in the sinc-collocation method for a boundary value problem with fourth-order ordinary differential equation. J. Comput. Appl. Math., 182 (2005), 32-50.

[10] A. Nurmuhammad, M. Muhammad and M. Mori, Sinc-Galerkin method based on the DE transformation for the boundary value problem of fourth-order ODE. J. Comput. Appl. Math., 206 (2007), 17-26.

[11] R.E. O'Malley Jr., Singular Perturbation Methods for Ordinary Differential Equations. Applied Mathematical Sciences, 89, Springer-Verlag, 1991.

[12] I. Radeka and D. Herceg, High-order methods for semilinear singularly perturbed boundary value problems. Novi Sad J. Math., 33 (2003), 139-161.

[13] S.M. Roberts, A boundary-value technique for singular perturbation problems. J. Math. Anal. Appl., 87 (1982), 489-508.

[14] H.-G. Roos, M. Stynes and L. Tobiska, Numerical Methods for Singularly Perturbed Differential Equations, Convection-Diffusion and Flow Problems. Springer Series in Computational Mathematics, 24, Springer 1996.

[15] F. Stenger, Numerical Methods Based on Sinc and Analytic Functions. Springer-Verlag, Berlin, New York, 1993.

[16] M. Sugihara, Optimality of the double exponential formula - functional analysis approach-. Numer. Math., 75 (1997), 379-395.

[17] M. Sugihara, Double exponential transformation in the sinc-collocation method for twopoint boundary value problems. J. Comput. Appl. Math., 149 (2002), 239-250.

[18] M. Sugihara, Near optimality of the sinc approximation. Math. Comput., 72 (2003), 767-786.

[19] H. Takahasi and M. Mori, Double exponential formulas for numerical integration. Publ. RIMS Kyoto Univ., 9 (1974), 721-741. 
